\title{
Модель двойной пористости для изучения разработки трециновато-пористых коллекторов на базе кониепиии суперэлементов
}

\author{
И.В. Афанаскин ${ }^{1}$, к.т.н., ведущий научный сотрудник, ivan@afanaskin.ru \\ С.Г. Вольпин ${ }^{1}$, к.т.н., зав. отделом, sergvolpin@gmail.com \\ A.B. Родителев ${ }^{1}$, ведущий программист, rav@niisi.ras.ru \\ A.A. Колеватов ${ }^{1}$, к.т.н., зам. зав. отделом, akolevatov@niisi.ras.ru \\ 1 Федеральный научный иентр Научно-исследовательский институт \\ системных исследований Российской академии наук, г. Москва, 117218, Россия
}

Основной технологией разработки нефтяных месторождений в России является заводнение (закачка в нефтяной пласт воды для вытеснения нефти и поддержания пластового давления). При этом большая часть нефтяных месторождений нашей страны находится на 3-й или 4-й стадии разработки, что означает высокую обводненность добываемой жидкости (90 \% и более). Основная задача специалистов по разработке нефтяных месторождений - уменьшить добычу воды и (по возможности) увеличить добычу нефти. В этих условиях много внимания уделяется контролю и регулированию разработки нефтяных месторождений.

Для выполнения этих работ специалистам необходим инструмент, позволяющий быстро строить модели значительных по размерам месторождений и оперативно рассчитывать большое количество сценариев для проверки гипотез о геологическом строении, адаптации модели и решения задач оптимизации разработки. Наиболее актуально это для трещиновато-пористых коллекторов, поскольку они характеризуются высокой неоднородностью фильтрационно-емкостных свойств. Это провоцирует опережающее обводнение добывающих скважин, что препятствует достижению проектных показателей разработки месторождений.

Предлагается методика численного математического моделирования разработки нефтяных месторождений в карбонатных трещиновато-поровых коллекторах на базе концепции суперэлементов. Фильтрация двухфазная, применена концепция двойной пористости. Численная схема полностью явная. Система уравнений сохранения аппроксимирована по пространству на суперэлементной сетке. Это позволяет существенно увеличить скорость вычислений и упростить построение моделей (так как размер ячеек сопоставим с расстоянием между скважинами). Для корректных расчетов необходима адаптация на историю разработки.

Предлагаемая методика тестируется на модели реального месторождения, результаты расчетов сравниваются с расчетами на коммерческом симуляторе Rubis Kappa Engineering. Получены хорошее совпадение на этапе обучения модели и удовлетворительное совпадение результатов прогнозных расчетов.

Ключевье слова: моделирование разработки нефтяных месторождений, двухфазная фильтрация, карбонатный коллектор, трещиновато-пористый коллектор, модель двойной пористости, суперэлементыл.

Разработка нефтяных месторождений в карбонатных трещиновато-пористых коллекторах сопряжена со значительными сложностями, поскольку такие коллекторы характеризуются большой неоднородностью фильтрационноемкостных свойств как по толщине, так и по площади. Кроме того, данные коллекторы состоят из двух сред с резко отличающимися характеристиками - из пор и трещин. Эти среды вложены друг в друга и обмениваются флюидами. Высокая проницаемость трещин и их низкая пористость (пустотность) по сравнению с порами приводят к опережающему обводнению добывающих скважин, что негативно сказывается на показателях разработки. Кроме того, в арсенале нефтяников практически отсутствуют средства, позволяющие с высокой точностью определять характеристики таких пластов. Проблема состоит в раздельном определении параметров пор и трещин. Большая часть имеющихся методов исследований пластов определяет интегральные характеристики, а остальные дают неоднозначный либо сложноинтерпретируемый результат. 
При задании фильтрационно-емкостных свойств и визуализации результатов моделирования трещиновато-пористых коллекторов обычно считается, что пласт содержит в два раза больше ячеек $(2 N)$. Тогда первая половина сетки (ячейки с 1-й по $N$-ю) описывает матрицу, а вторая (ячейки с $N+1-$ й по $2 N$-ю) - трещины. Каждой ячейке матрицы должна соответствовать своя активная ячейка трещины и наоборот. Это двухкратное увеличение количества расчетных ячеек сильно замедляет расчеты.

В таких условиях актуальным является создание инструмента для оперативного моделирования разработки трещиновато-пористых коллекторов, позволяющего быстро прогонять большое количество расчетных вариантов как для адаптации модели и уточнения параметров пласта, так и для оптимизации показателей разработки. Настоящая работа посвящена такой модели, основанной на концепции суперэлементов.

\section{Математическая модель}

Введем физико-математическую модель двойной пористости при следующих условиях:

- осуществляется двухфазная фильтрация нефти и воды при забойном давлении, превышающем либо равном давлению насыщения пластовой нефти газом;

- жидкости слабосжимаемые, пласт упругий;

- пласт состоит из двух вложенных друг в друга сред - пористых блоков (матрицы) и трещин; пористые блоки разбиты трещинами регулярным образом на параллелепипеды;

- проницаемость трещин намного больше проницаемости матрицы; пористость (пустотность) трещин намного меньше пористости матрицы;

- в скважине работают только трещины;

- трещины обмениваются жидкостью с пористой матрицей, переток жидкости псевдоустановившийся;

- фильтрацией жидкости внутри матрицы пренебрегаем.

Тогда можно записать две системы уравнений сохранения - отдельно для трещин и для матрицы. Эти системы будут связаны между собой источниковыми слагаемыми, отвечающими за переток фаз из одной среды в другую [1].
Уравнения сохранения объемов нефти и воды в стандартных условиях в трещинах запишем в виде:

$$
\begin{aligned}
& \frac{\partial}{\partial t}\left(\frac{\phi_{f} S_{o, f}}{B_{o, f}}\right)+\nabla\left(\frac{\vec{W}_{o, f}}{B_{o, f}}\right)=-\bar{q}_{o}+\bar{q}_{o, m f}, \\
& \frac{\partial}{\partial t}\left(\frac{\phi_{f} S_{w, f}}{B_{w, f}}\right)+\nabla\left(\frac{\vec{W}_{w, f}}{B_{w, f}}\right)=-\bar{q}_{w}+\bar{q}_{w, m f} ;
\end{aligned}
$$

уравнения сохранения количества движения для трещин запишем в виде обобщенного закона Дарси:

$$
\begin{aligned}
& \vec{W}_{o, f}=-\frac{k_{f} k_{r o, f}}{\mu_{o}}\left[\nabla\left(P_{o, f}\right)-\rho_{o, f} g \nabla\left(D_{f}\right)\right], \\
& \vec{W}_{w, f}=-\frac{k_{f} k_{r w, f}}{\mu_{w}}\left[\nabla\left(P_{w, f}\right)-\rho_{w, f} g \nabla\left(D_{f}\right)\right],
\end{aligned}
$$

где $\phi_{f}$ и $k_{f}-$ пористость и абсолютная проницаемость трещин; $\bar{q}_{o, m f}$ и $\bar{q}_{w, m f}$ - плотность перетока нефти и воды из матрицы в трещины; $S_{o, f}$ и $S_{w, f}, B_{o, f}$ и $B_{w, f}, \vec{W}_{o, f}$ и $\vec{W}_{w, f}, \bar{q}_{o}$ и $\bar{q}_{w}, k_{r o, f}$ и $k_{r w, f}, P_{o, f}$ и $P_{w, f}, \rho_{o, f}$ и $\rho_{w, f}-$ насыщенность, объемный коэффициент, вектор скорости фильтрации, плотность источника (стока), моделирующего работу скважины, относительная фазовая проницаемость (ОФП), давление и плотность в пластовых условиях нефти и воды в трещинах; $\mu_{o}$ и $\mu_{w}-$ вязкость нефти и воды; $g$ - ускорение свободного падения; $D_{f}-$ глубина залегания трещины по вертикали [1-3].

Система уравнений (1)-(4) дополняется замыкающими соотношениями следующим образом:

$$
\begin{aligned}
& S_{o, f}+S_{w, f}=1, \\
& \phi_{f}=\phi_{f, 0}\left[1+C_{r, f}\left(P_{f}-P_{f, 0}\right)\right], \\
& B_{\alpha}=B_{\alpha, 0}\left[1-C_{\alpha}\left(P-P_{0}\right)\right], \alpha=o, w, \\
& \rho_{\alpha}=\rho_{\alpha, S T C} / B_{\alpha}, \alpha=o, w, \\
& P_{o, f}-P_{w, f}=P_{c, f}\left(S_{w, f}\right),
\end{aligned}
$$

где $\phi_{f, 0}$ - пористость трещин при начальном пластовом давлении $P_{f, 0} ; B_{\alpha, 0}, \alpha=o, w$ - объемный коэффициент фазы $\alpha$ при начальном пластовом давлении; $C_{r, f}, C_{o, f}$ и $C_{w, f}-$ сжимаемость трещин, нефти и воды; $\rho_{\alpha}, \alpha=o, w$ - плотность фазы $\alpha ; \rho_{\alpha, S T C}, \alpha=o, w-$ плотность фазы $\alpha$ в стандартных условиях; $P_{c, f}-$ капиллярное давление в трещинах в системе нефть-вода.

Систему уравнений (1)-(4) с учетом соотношений (5)-(9), принимая $S_{f} \equiv S_{w, f}, P_{f} \equiv P_{o, f}$ и используя подход, изложенный в [4], можно 
преобразовать в следующую систему уравнений:

$$
\begin{gathered}
\phi_{f, 0}\left[C_{r, f}+\left(C_{w}-C_{o}\right) S_{f}+C_{o}\right] \frac{\partial P_{f}}{\partial t}+ \\
+B_{o 0} \nabla\left(\frac{\vec{W}_{o, f}}{B_{o, f}}\right)+B_{w, 0} \nabla\left(\frac{\vec{W}_{w, f}}{B_{w, f}}\right)= \\
=-B_{o 0}\left[\bar{q}_{o}-\bar{q}_{o, m f}\right]-B_{w, 0}\left[\bar{q}_{w}-\bar{q}_{w, m f}\right], \\
\phi_{f, 0}\left[\frac{\partial S_{f}}{\partial t}+\left(C_{r, f}+C_{w}\right) S_{f} \frac{\partial P_{f}}{\partial t}\right]+ \\
+B_{w, 0} \nabla\left(\frac{\vec{W}_{w, f}}{B_{w, f}}\right)=-B_{w, 0}\left[\bar{q}_{w}-\bar{q}_{w, m f}\right], \\
\vec{W}_{o, f}=-\frac{k_{f} k_{r o, f}}{\mu_{o}}\left[\nabla\left(P_{f}\right)-\rho_{o, f} g \nabla\left(D_{f}\right)\right], \\
\vec{W}_{w, f}=-\frac{k_{f} k_{r w, f}}{\mu_{w}}\left[\nabla\left(P_{f}\right)-\nabla\left(P_{c, f}\right)-\rho_{w, f} g \nabla\left(D_{f}\right)\right] .
\end{gathered}
$$

Система уравнений (10)-(13) дополняется начальными условиями следующим образом:

$P_{f}=P_{f}(x, y, z, t=0), S_{f}=S_{f}(x, y, z, t=0)$

и граничными условиями непротекания на внешних границах. Внутренние граничные условия на скважинах учитываются с помощью слагаемых $\bar{q}_{o, f}$ и $\bar{q}_{w, f}$.

Уравнения сохранения объемов нефти и воды в стандартных условиях в порах запишем в виде:

$$
\begin{aligned}
& \frac{\partial}{\partial t}\left(\frac{\phi_{m} S_{o, m}}{B_{o, m}}\right)=-\bar{q}_{o, m f}, \\
& \frac{\partial}{\partial t}\left(\frac{\phi_{m} S_{w, m}}{B_{w, m}}\right)=-\bar{q}_{w, m f},
\end{aligned}
$$

где обозначения аналогичны обозначениям, принятым для системы (1)-(2), с точностью до нижнего индекса $m$, обозначающего матричные блоки; уравнения сохранения количества движения для пор отсутствуют, так как фильтрацией жидкости в пористой матрице пренебрегаем [1-3].

Система уравнений (14)-(15) дополняется замыкающими соотношениями:

$$
\begin{aligned}
& S_{o, m}+S_{w, m}=1, \\
& \phi_{m}=\phi_{m, 0}\left[1+C_{r, m}\left(P_{m}-P_{m, 0}\right)\right], \\
& P_{o, m}-P_{w, m}=P_{c, m}\left(S_{w, m}\right),
\end{aligned}
$$

где $\phi_{m, 0}-$ пористость матрицы при начальном пластовом давлении $P_{m, 0} ; C_{r, m}-$ сжимаемость пор; $P_{c, m}-$ капиллярное давление в порах в системе нефть-вода.

Систему уравнений (14)-(15) с учетом соотношений (16)-(18), принимая $S_{m} \equiv S_{w, m}$, $P_{m} \equiv P_{o, m}$ и используя подход, изложенный в [4], можно преобразовать к следующей системе уравнений:

$$
\begin{aligned}
& \phi_{m, 0}\left[C_{r, m}+\left(C_{w}-C_{o}\right) S_{m}+C_{o}\right] \frac{\partial P_{m}}{\partial t}= \\
& =-B_{o 0} \bar{q}_{o, m f}-B_{w, 0} \bar{q}_{w, m f}, \\
& \phi_{m, 0}\left[\frac{\partial S_{m}}{\partial t}+\left(C_{r, m}+C_{w}\right) S_{m} \frac{\partial P_{m}}{\partial t}\right]=-B_{w, 0} \bar{q}_{w, m f} .
\end{aligned}
$$

Система уравнений (19)-(20) дополняется начальными условиями следующим образом:

$$
P_{m}=P_{m}(x, y, z, t=0), S_{m}=S_{m}(x, y, z, t=0)
$$

и граничными условиями непротекания на внешних границах.

Перетоки нефти и воды между трещинами и пористой матрицей учитываются с помощью слагаемых $\bar{q}_{o, m f}$ и $\bar{q}_{w, m f}$. Эти слагаемые связывают системы уравнений (10)-(13) и (19)-(20).

\section{Численная схема}

Аппроксимируем уравнения (10)-(13) и (19)-(20) на суперэлементной расчетной сетке [5, 6]. Размеры ячеек сопоставимы с расстоянием между скважинами (300-500 м). Сами ячейки строятся вокруг скважин. Тогда количество ячеек в одном горизонтальном слое примерно равно количеству скважин (иногда в больших неразбуренных областях вводятся фиктивные скважины для сгущения сетки). Форма ячеек соответствует сетке Вороного. Благодаря малому числу ячеек и их большому размеру такая вычислительная сетка позволяет использовать полностью явную расчетную схему и проводить расчеты с большой скоростью.

Суперэлементная сетка в горизонтальной плоскости близка к разбиению залежи на зоны дренирования по методу А.П. Крылова [7]. Выделяют четыре этапа построения суперэлементной сетки в плане:

- отображение на карте границ залежи и скважин;

- объединение внешних скважин и построение по ним выпуклой оболочки;

- проведение триангуляции Делоне по скважинам;

- отображение элементов Вороного, опус- 
кая перпендикуляры из середин ребер построенной триангуляции.

Для построения трехмерной сетки суперэлементов двухмерные горизонтальные слои копируют и размещают друг под другом вдоль вертикальной оси, изменяя при этом толщину слоев в соответствии с геологическими представлениями о строении пласта. Слои залегают друг под другом без разрывов между ними. Однако можно вводить слои с полным или частичным отсутствием коллектора для моделирования строения реальных месторождений.

Рассмотрим двухмерную (для упрощения записи) полностью явную (по давлению и насыщенности) численную схему в горизонтальной плоскости для уравнений (10)-(13) и (19)-(20) и выразим неизвестные:

$\left(P_{f}\right)_{i}^{n+1}=\left(P_{f}\right)_{i}^{n}-$

$-\Delta t^{n+1} \frac{\sum_{\alpha=o, w}\left(B_{\alpha, 0}\left\{\left(q_{\alpha}\right)_{i}^{n}-\left(q_{\alpha, m f}\right)_{i}^{n}+\sum_{j=1}^{N_{i}}\left[A_{i j} h_{i j}\left(\frac{W_{\alpha}}{B_{\alpha}}\right)_{i j}^{n}\right]\right\}\right)}{F_{i} h_{i}\left(\phi_{f, 0}\right)_{i}\left[C_{r, f}+\left(C_{w}-C_{o}\right)\left(S_{f}\right)_{i}^{n}+C_{o}\right]}$

$\left(S_{f}\right)_{i}^{n+1}=\left(S_{f}\right)_{i}^{n}-$

$-\Delta t^{n+1} \frac{B_{w, 0}}{F_{i} h_{i}\left(\phi_{f, 0}\right)_{i}}\left\{\left(q_{w}\right)_{i}^{n}-\left(q_{w, n f}\right)_{i}^{n}+\sum_{j=1}^{N_{i}}\left[A_{i j} h_{i j}\left(\frac{W_{w}}{B_{w}}\right)_{i j}^{n}\right]\right\}$

$-\left(C_{r, f}+C_{w}\right)\left(S_{f}\right)_{i}^{n}\left[\left(P_{f}\right)_{i}^{n+1}-\left(P_{f}\right)_{i}^{n}\right]$,

$\left(P_{m}\right)_{i}^{n+1}=\left(P_{m}\right)_{i}^{n}-$

$-\Delta t^{n+1} \frac{B_{o, 0}\left(q_{o, m f}\right)_{i}^{n}+B_{w, 0}\left(q_{w, m f}\right)_{i}^{n}}{F_{i} h_{i}\left(\phi_{m, 0}\right)_{i}\left[C_{r, m}+\left(C_{w}-C_{o}\right)\left(S_{m}\right)_{i}^{n}+C_{o}\right]}$,

$\left(S_{m}\right)_{i}^{n+1}=\left(S_{m}\right)_{i}^{n}-\Delta t^{n+1} \frac{B_{w, 0}}{F_{i} h_{i}\left(\phi_{m, 0}\right)_{i}}\left(q_{w, m f}\right)_{i}^{n}-$

$-\left(C_{r, m}+C_{w}\right)\left(S_{m}\right)_{i}^{n}\left[\left(P_{m}\right)_{i}^{n+1}-\left(P_{m}\right)_{i}^{n}\right]$,

где нижний индекс $i-$ номер ячейки; верхний индекс $n$ - номер шага по времени; $\Delta t^{n+1}-$ переменный шаг по времени (определяется исходя из ограничения на максимальное изменение давления и насыщенности за один временной шаг $[2,8]) ; A_{i j}$ - длина ребра между соседними ячейками $i$ и $j ; h_{i}-$ толщина ячейки $i ; h_{i j}$ - средняя толщина соседних ячеек $i$ и $j$ (определяется как среднее арифметическое); $N_{i}$ - количество соседних ячеек для ячейки $i$; $\left(B_{\alpha}\right)_{i j}^{n}$ - средний объемный коэффициент фазы $\alpha=o, w$ между соседними ячейками $i$ и $j$ (определяется как среднее арифметическое); $\left(W_{\alpha}\right)_{i j}^{n}$
- скорость фильтрации в трещинах фазы $\alpha=o$, $w$ между соседними ячейками $i$ и $j$ :

$$
\begin{aligned}
& \left(W_{\alpha}\right)_{i j}^{n}=\frac{k_{i j}\left(k_{r \alpha}\right)_{i j}^{n}}{\mu_{\alpha}} \cdot \frac{\left(\Phi_{\alpha, f}\right)_{i}^{n}-\left(\Phi_{\alpha, f}\right)_{j}^{n}}{L_{i j}}, \alpha=o, w, \\
& k_{i j}=\frac{2\left(k_{f}\right)_{i}\left(k_{f}\right)_{j}}{\left(k_{f}\right)_{i}+\left(k_{f}\right)_{j}}, \\
& \left(k_{r \alpha}\right)_{i j}^{n}=\left\{\begin{array}{l}
\left(k_{r \alpha, f}\right)_{i}^{n},\left(\Phi_{\alpha, f}\right)_{i}^{n} \geq\left(\Phi_{\alpha, f}\right)_{j}^{n}, \\
\left(k_{r \alpha, f}\right)_{j}^{n},\left(\Phi_{\alpha, f}\right)_{i}^{n}<\left(\Phi_{\alpha, f}\right)_{j}^{n},
\end{array}\right. \\
& \left(\Phi_{o, f}\right)_{i}^{n}=\left(P_{f}\right)_{i}^{n}-\left(\rho_{o, f}\right)_{i}^{n} g\left(D_{f}\right)_{i}, \\
& \left(\Phi_{w, f}\right)_{i}^{n}=\left(P_{f}\right)_{i}^{n}-\left(P_{c, f}\right)_{i}^{n}-\left(\rho_{w, f}\right)_{i}^{n} g\left(D_{f}\right)_{i},
\end{aligned}
$$

где $L_{i j}$ - расстояние между центрами двух соседних ячеек.

Скважины моделируются следующим образом. Необходимо задать либо один из дебитов (жидкости, нефти или воды), либо забойное давление. Остальные параметры рассчитываются. Например, при заданном дебите жидкости $\left(q_{l}\right)_{i}^{n}$ имеем забойное давление $\left(P_{\text {well }}\right)_{i}^{n}$, которое рассчитывается следующим образом:

$$
\left(P_{\text {well }}\right)_{i}^{n}=\left(P_{f}\right)_{i}^{n}-\left(q_{l}\right)_{i}^{n} /\left\{W I_{i}^{n}\left[\left(\lambda_{o, f}\right)_{i}^{n}+\left(\lambda_{w, f}\right)_{i}^{n}\right]\right\},
$$

где $W I_{i}^{n}=\frac{2 \pi\left(k_{f}\right)_{i} h_{i}}{\ln \left[\frac{\left(R_{c}\right)_{i}}{\left(r_{w}\right)_{i}}\right]+S k i n_{i}^{n}} ; \quad\left(R_{c}\right)_{i}=0,14 \sqrt{2 F_{i}}$; $\left(\lambda_{\alpha, f}\right)_{i}^{n}=\frac{\left(k_{r \alpha, f}\right)_{i}^{n}}{\mu_{\alpha}\left(B_{\alpha, f}\right)_{i}^{n}}, \alpha=o, w ;\left(\lambda_{\alpha, f}\right)_{i}^{n}-$ подвижность фазы $\alpha=o, w$ в трещинах; $W I_{i}^{n}-$ коэффициент продуктивности скважины; $\left(R_{c}\right)_{i}-$ радиус контура питания; $\left(r_{w}\right)_{i}$ - радиус скважины; Skin $_{i}^{n}$ - скин-фактор скважины.

После определения забойного давления $\left(P_{\text {well }}\right)_{i}^{n}$ дебиты по фазам определяются как

$$
\left(q_{\alpha}\right)_{i}^{n}=W I_{i}^{n}\left(\lambda_{\alpha, f}\right)_{i}^{n}\left[\left(P_{f}\right)_{i}^{n}-\left(P_{\text {well }}\right)_{i}^{n}\right], \alpha=o, w .
$$

Переток фаз между матрицей и трещинами $\left(q_{\alpha, m f}\right)_{i}^{n}, \alpha=o, w$, осуществляется в псевдоустановившемся режиме и определяется формулой (по аналогии с приводимыми в [1])

$$
\left(q_{\alpha, m f}\right)_{i}^{n}=\left(T_{m f}\right)_{i} \frac{\left(k_{r \alpha, m f}\right)_{i}^{n}}{\mu_{\alpha}\left(B_{\alpha, m f}\right)_{i}^{n}}\left[\left(\Phi_{\alpha, m}\right)_{i}^{n}-\left(\Phi_{\alpha, f}\right)_{i}^{n}\right] F_{i} h_{i},
$$

$\alpha=o, w$, 
где $\left(T_{m f}\right)_{i}=C_{D}\left(k_{m f}\right)_{i} \sigma_{i}, \sigma_{i}=4\left(\frac{1}{l_{x}^{2}}+\frac{1}{l_{y}^{2}}+\frac{1}{l_{z}^{2}}\right)$,

$\left(k_{m f}\right)_{i}=\frac{2\left(k_{f}\right)_{i}\left(k_{m}\right)_{i}}{\left(k_{f}\right)_{i}+\left(k_{m}\right)_{i}}$

$\left(k_{r \alpha, m f}\right)_{i}^{n}=\left\{\begin{array}{l}\left(k_{r \alpha, m}\right)_{i}^{n},\left(\Phi_{\alpha, m}\right)_{i}^{n} \geq\left(\Phi_{\alpha, f}\right)_{i}^{n}, \\ \left(k_{r \alpha, f}\right)_{i}^{n},\left(\Phi_{\alpha, m}\right)_{i}^{n}<\left(\Phi_{\alpha, f}\right)_{i}^{n},\end{array}\right.$

$\left(\Phi_{o, m}\right)_{i}^{n}=\left(P_{m}\right)_{i}^{n}-\left(\rho_{o, m}\right)_{i}^{n} g\left(D_{m}\right)_{i}$,

$\left(\Phi_{w, m}\right)_{i}^{n}=\left(P_{m}\right)_{i}^{n}-\left(P_{c, m}\right)_{i}^{n}-\left(\rho_{w, m}\right)_{i}^{n} g\left(D_{m}\right)_{i}$,

$\left(T_{m f}\right)_{i}$ - матрично-трещинная проводимость;

$C_{D}-$ коэффициент Дарси; $\sigma_{i}-$ фактор формы (шейп-фактор); $l_{x}, l_{y}$ и $l_{z}$ - размеры блока матрицы; $\left(B_{\alpha, m f}\right)_{i}^{n}-$ средний объемный коэффициент фазы $\alpha=o, w$ между матрицей и трещинами (определяется как среднее арифметическое).

В такой модели вода появится в продукции добывающей скважины сразу же после того, как текущая водонасыщенность ячейки превысит значение насыщенности связанной водой. В реальности этого не происходит. Воде необходимо время для того, чтобы пройти расстояние от нагнетательной скважины до добывающей. Для воссоздания этого эффекта используется ступенчатое задание функций относительной фазовой проницаемости: если водонасыщенность ячейки меньше насыщенности связанной водой, то ОФП по воде равна нулю, а ОФП по нефти равна своему максимальному значению; если водонасыщенность ячейки больше насыщенности связанной водой, то ОФП по нефти и воде рассчитываются как функции водонасыщенности $[5,6,9]$.

Соотношения (21)-(24) позволяют рассчитывать давление и насыщенность в порах и трещинах. Они могут быть легко обобщены на трехмерный случай.

\section{Моделирование реального месторождения}

Рассмотрим применение предлагаемой модели двойной пористости для изучения разработки трещиновато-пористых коллекторов на базе концепции суперэлементов на примере реального нефтяного месторождения.

Продуктивный пласт представлен трещиновато-пористыми карбонатными породами мелового периода. Залежь нефти пластового типа, литологически ограниченная. Перечислим ос- новные геолого-физические параметры пласта.

- Средняя проницаемость трещин 1600 Д, пористость трещин - 0,04 д.ед.

- Средняя проницаемость матрицы 1,3 мД, пористость матрицы - 0,22 д.ед.

- Эффективная нефтенасыщенная толщина -8 м.

- Начальное пластовое давление - 339 бар, начальная пластовая температура $-147^{\circ} \mathrm{C}$.

- Сжимаемость трещин - 1,5·10-4 1/бар, сжимаемость пористых блоков $-4,5 \cdot 10^{-5} 1 /$ бар.

- PVT-свойства воды: объемный коэффициент $-1,02 \mathrm{~m}^{3} / \mathrm{M}^{3}$, вязкость $-0,36 \mathrm{мПа} с$, сжимаемость $-4,7 \cdot 10^{-5} 1 / 6 а р$, плотность в стандарт-

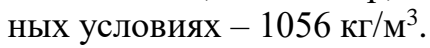

- PVT-свойства нефти: объемный коэффициент $-1,55 \mathrm{~m}^{3} / \mathrm{M}^{3}$, вязкость $-0,397 \mathrm{мПа} \cdot \mathbf{c}$, сжимаемость $-1,01 \cdot 10^{-4} 1 / 6 а р$, плотность в стандартных условиях - 815 кг/м³ щения нефти газом - 188 бар, растворимость газа в нефти $-203 \mathrm{~m}^{3} / \mathrm{M}^{3}$.

ОФП и капиллярные давления в системе нефть-вода приведены на графиках (рис. 1).

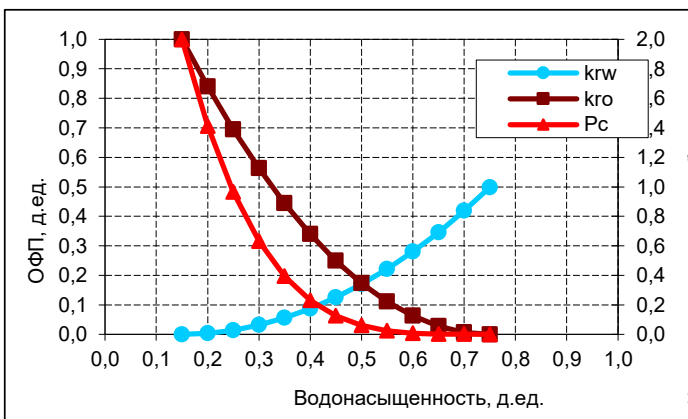

a)

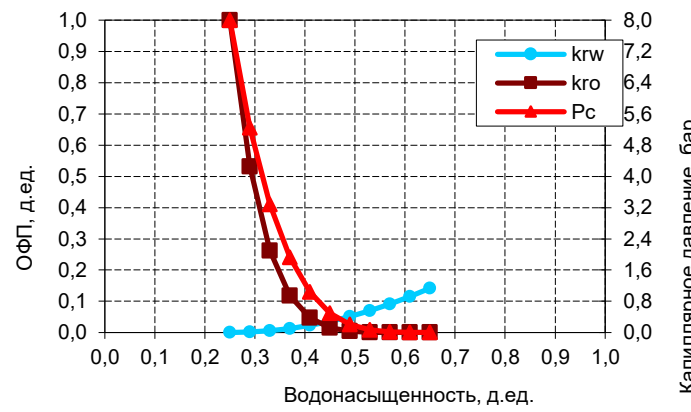

б)

Рис. 1. ОФП для нефти (kro) и воды (krw), а также капиллярное давление $(P c)$ в системе нефть-вода: а) для трещин, б) для пористой матрицьь

Fig. 1. Relative permeability for oil (kro) and water (krw), as well as capillary pressure $(P c)$ in the oil-water system: a) for fractures, б) for a porous matrix 
Всего в разработке перебывало 127 скважин, из них 29 нагнетательных и 98 добывающих.

Для проверки применимости предлагаемой модели двойной пористости были созданы две гидродинамические модели: одна с помощью предлагаемой модели, другая - коммерческого симулятора Rubis компании Kappa Engineering [10]. Построенные вычислительные сетки приведены на рисунках (cм. http://www.swsys. ru/uploaded/image/2019-3/2019-3-dop/1.jpg, http://www.swsys.ru/uploaded/image/2019-3/ 2019-3-dop/2.jpg). Для сгущения сетки при построении суперэлементной модели были использованы 30 фиктивных скважин (на рисунке они названы пустыми). История разра- ботки месторождения - 33 года. Из них 26 лет занял этап обучения суперэлементной модели, а последующие 7 лет - прогноз. Расчеты проводились при задаваемых на скважинах фактически замеренных дебитах добываемой жидкости и расходах закачиваемой воды. В процессе обучения модели уточнялись относительные фазовые проницаемости, проницаемость матрицы и пористость трещин. Суточная и накопленная добыча нефти и воды показаны на рисунках 2 и 3 . Получено хорошее совмещение кривых, рассчитанных с помощью суперэлементной модели и Rubis, на периоде обучения суперэлементной модели и удовлетворительное совмещение на периоде прогноза.

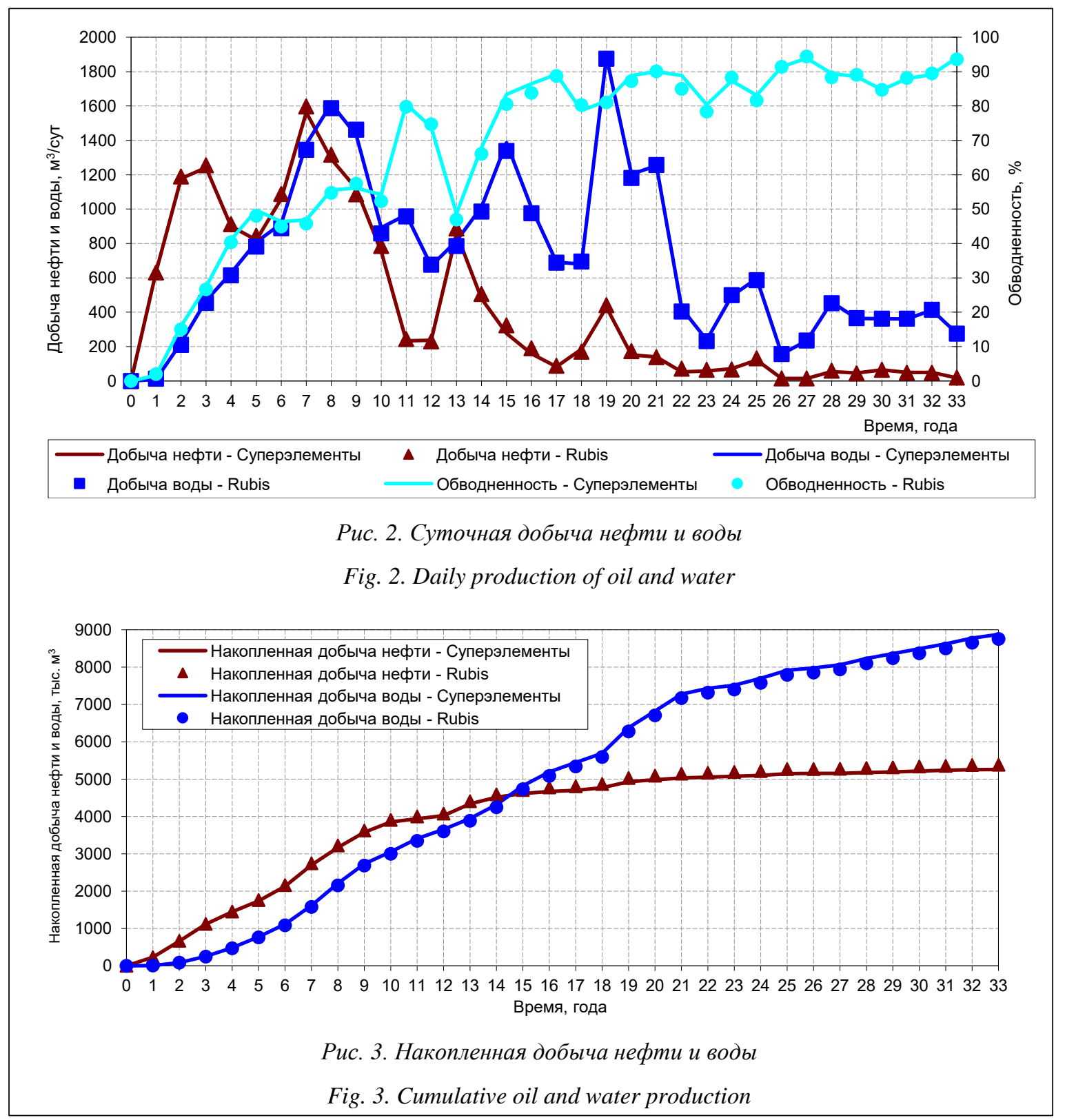




\section{Заключение}

В работе описана модель двойной пористости для изучения разработки трещиновато-пористых коллекторов на базе концепции суперэлементов. Фильтрация двухфазная при забойном давлении в добывающих скважинах выше давления насыщения. Модель предполагает отсутствие фильтрации флюида по матрице. Приток жидкости в ствол скважины только из трещин, переток между матрицей и трещинами в псевдоустановившемся режиме. Размер ячеек суперэлементной сетки сопоставим с расстоянием между скважинами. Благодаря этому использована полностью явная численная схема (по давлению и насыщенности) с переменным шагом по времени. Болышеразмерные ячейки позволяют ис- пользовать упрощенные геологические представления и быстро рассчитывать даже модели с очень большим количеством скважин. Это дает возможность применения предлагаемой модели для многовариантных расчетов и решения оптимизационных задач. Из-за больших размеров сетки обязательным этапом является адаптация модели на историю разработки или обучение модели с использованием результатов расчетов на более мелкой сетке. Предлагаемая математическая модель апробирована на реальном месторождении, результаты расчетов сравниваются с вычислениями с помощью коммерческого симулятора Rubis Kappa Engineering. Получены хорошее совпадение показателей разработки на этапе обучения модели и удовлетворительное совпадение на этапе прогноза

Работа выполнена при поддержке РФФИ, грант № 16-29-15135 офи_м.

\section{Лuтература}

1. Голф-Рахт Т.Д. Основы нефтепромысловой геологии и разработки трещиноватых коллекторов. М.: Недра, 1986. 608 с.

2. Азиз Х., Сеттари Э. Математическое моделирование пластовых систем. М.-Ижевск: Изд-во ИКИ, 2004. 416 с.

3. Каневская Р.Д. Математическое моделирование гидродинамических процессов разработки месторождений углеводородов. М.-Ижевск: Изд-во ИКИ, 2002. 140 с.

4. Кац Р.М., Волгин Е.Р., Афанаскин И.В. Численное моделирование двухфазной фильтрации нефти и воды // Тр. НИИСИ РАН. 2014. Т. 4. № 2. С. 141-148.

5. Булыгин Д.В., Мазо А.Б., Поташев К.А., Калинин Е.И. Геолого-технические аспекты суперэлементной фильтрационной модели нефтяных месторождений // Георесурсы. 2013. № 3. С. 31-35.

6. Мазо А.Б., Булыгин Д.В. Суперэлементы. Новый подход к моделированию разработки нефтяных месторождений // Нефть. Газ. Новации. 2011. № 11. С. 6-8.

7. Хисамутдинов Н.И., Хасанов М.М., Телин А.Г., Ибрагимов Г.З., Латыпов А.З., Потапов А.М. Разработка нефтяных месторождений: в 4 т.; Т. 1. Разработка нефтяных месторождений на поздней стадии. М.: Изд-во ВНИИОЭНГ, 1994. 240 с.

8. Эртекин Т., Абу-Кассем Дж., Кинг Г. Основы прикладного моделирования. Ижевск: Изд-во ИКИ, 2012. 1060 с.

9. Афанаскин И.В., Ялов П.В., Гиацинтов А.М., Родителев А.В. Решение задач оптимизации при суперэлементном моделировании разработки нефтяных месторождений // Программные продукты и системы. 2017. Т. 30. № 3. С. 384-391. DOI: 10.15827/0236-235X.119.384-391.

10. Houze O., Viturat D., Fjaere O.S. Dynamic Data Analysis. V 5.20.01. Kappa Engineering Publ., 2018, 757 p.

\section{Dual porosity model for fractured porous reservoirs development analysis based on the superelement concept}

I.V.Afanaskin ${ }^{1}$, Ph.D. (Engineering), Leading Researcher, ivan@afanaskin.ru

S.G. Volpin ${ }^{1}$, Ph.D. (Engineering), Head of Department, sergvolpin@gmail.com

A.V.Roditelev ${ }^{1}$, Leading Programmer, rav@niisi.ras.ru

A.A. Kolevatov ${ }^{1}$, Ph.D. (Engineering), Deputy Head of Department, akolevatov@niisi.ras.ru

${ }^{1}$ Federal State Institution "Scientific Research Institute for System Analysis of the Russian Academy of Sciences" (SRISA RAS), Moscow, 117218, Russian Federation

Abstract. The main oil field development strategy in Russia is waterflooding (water injection into an oil reservoir for oil displacement and pressure maintenance). Nowadays, most Russian oil fields are at the 3rd and 
4th development stages, which means high water cut of produced liquid (90\% and more). Main objective of reservoir engineers is water production reduction (if possible) and oil production increase. These conditions require significant control and oil reservoir development regulation.

To implement such activities, specialists need a solution for fast simulation of significant reservoirs and for fast evaluation of multiple development scenarios for testing hypothesis for geological structure, history matching and production optimization. This approach is relevant for fractured porous reservoirs that have significant heterogeneity of filtration-conductivity properties. This fact causes early watercut growth in producing wells and leads to limitation of field production project targets.

The paper proposes a methodology of numerical simulation of fractured porous oil reservoirs development based on the superelement conception. The model simulates two-phase filtration in a dual-porosity reservoir. A numerical scheme is fully explicit. The set of conservation equations is approximated on a super-elements grid. This fact increases calculation speed and simplifies model generation (as cell dimension is consistent with well spacing). Calculation accuracy check requires production history matching.

The proposed calculation methodology is tested on a real field example and checked by simulation in Rubis Kappa Engineering. Good matching results have been achieved at model training stages and forecast simulation.

Keywords: oil field development simulation, two-phase filtration, carbonate reservoir, fractured porous reservoir, dual-porosity filtration mechanism, super-elements.

Acknowledgements. The paper has been supported by the program of Russian Foundation for Basic Research no. 16-29-15135 ofi_m.

\section{References}

1. Golf-Raht T.D. Fundamentals of Oilfield Geology and Fractured Reservoir Development. Moscow, Nedra Publ., 1986, 608 p.

2. Aziz Kh., Settari A. Petroleum Reservoir Simulation. London, Applied Science Publ., 1979, 476 p. (Russ. ed.: Moscow-Izhevsk, ICS Publ., 2004, 416 p.).

3. Kanevskaya R.D. Mathematical Modeling of Hydrodynamic Processes of Hydrocarbon Deposit Development. Moscow-Izhevsk, ICS Publ., 2002, 140 p.

4. Kats R.M., Volgin E.R., Afanaskin I.V. Numerical simulation of two-phase oil and water filtration. SRISA RAS Proc. 2014, vol. 4, iss. 2, pp. 141-148 (in Russ.).

5. Bulygin D.V., Mazo A.B., Potashev K.A., Kalinin E.I. Geological and technical aspects of a superelement filtration model of oil fields. Georesources. 2013, iss. 3, pp. 31-35 (in Russ.).

6. Mazo A.B., Bulygin D.V. Superelements. A new approach to modeling the development of oil fields. Oil. Gas. Novations. 2011, iss. 11, pp. 6-8 (in Russ.).

7. Khisamutdinov N.I., Khasanov M.M., Telin A.G., Ibragimov G.Z., Latypov A.Z., Potapov A.M. Development of Oil Fields. Vol. 1. Development of Oil Fields at the Late Stage. Moscow, VNIIOENG Publ., 1994, $240 \mathrm{p}$.

8. Ertekin T., Abu-Kassem J., King G. Basic Applied Reservoir Simulation. Izhevsk, ICS Publ., 2012, $1060 \mathrm{p}$.

9. Afanaskin I.V., Yalov P.V., Giatsintov A.M., Roditelev A.V. Optimization problems solution based on super-element modeling of oil-field development. Software \& Systems. 2017, vol. 30, no. 3, pp. 384-391. DOI: 10.15827/0236-235X.119.384-391 (in Russ.).

10. Houze O., Viturat D., Fjaere O.S. Dynamic Data Analysis. V 5.20.01. Kappa Engineering Publ., 2018, 757 p.

\section{Для цитирования}

Афанаскин И.В., Вольпин С.Г., Родителев А.В., Колеватов А.А. Модель двойной пористости для изучения разработки трещиновато-пористых комлекторов на базе концепции суперэлементов // Программные продукты и системы. 2019. Т. 32. № 3. С. 478-485. DOI: 10.15827/0236235X.127.478-485.

\section{For citation}

Afanaskin I.V., Volpin S.G., Roditelev A.V., Kolevatov A.A. Dual porosity model for fractured porous reservoirs development analysis based on the superelement concept. Software \& Systems. 2019, vol. 32, no. 3, pp. 478-485 (in Russ.). DOI: 10.15827/0236-235X.127.478-485. 